\title{
Erratum to: Correlation of electrode position and clinical outcomes in Globus pallidus stimulation for dystonia
}

\author{
Hye Ran Park ${ }^{1}$. Jae Min Lee ${ }^{2}$. Gwanhee Ehm ${ }^{3}$ - Hui-Jun Yang ${ }^{4}$ In Ho Song ${ }^{5}$. \\ Yong Hoon Lim ${ }^{2}$ Mi-Ryoung Kim² ${ }^{2}$ Keyoung Ran Kim ${ }^{2}$. Woong-Woo Lee ${ }^{6}$. \\ Young Eun Kim ${ }^{7}$. Jae Ha Hwang ${ }^{8}$. Chae Won Shin ${ }^{9}$. Hyeyoung Park ${ }^{9}$. \\ Jin Wook Kim ${ }^{2}$ - Han-Joon Kim ${ }^{9}$. Cheolyoung Kim ${ }^{10}$ • Dong Gyu Kim ${ }^{2,11}$. \\ Beom Seok Jeon ${ }^{9,12} \cdot$ Sun Ha Paek ${ }^{2,11}$
}

Published online: 10 May 2017

(C) Springer-Verlag Wien 2017

\section{Erratum to: Acta Neurochir}

DOI 10.1007/s00701-017-3182-4

The original version unfortunately contains error in texts in

Fig. 1. The correct Fig. 1 should be the below:

The online version of the original article can be found at http://dx.doi.org/ 10.1007/s00701-017-3182-4

Sun Ha Paek

paeksh@snu.ac.kr

1 Department of Neurosurgery, Soonchunhyang University Seoul Hospital, Seoul, Republic of Korea

2 Department of Neurosurgery, Seoul National University Hospital, Seoul, Republic of Korea

3 Department of Neurology, Myongji Hospital, Goyang, Gyeonggi, Republic of Korea

4 Department of Neurology, Ulsan University Hospital, Ulsan, Republic of Korea

5 Medical Device Development Center, Osong Medical Innovation Foundation, Boryeong, Chungcheong, Republic of Korea
Department of Neurology, Eulji General Hospital, Seoul, Republic of Korea

7 Department of Neurology, Hallym University Sacred Heart Hospital, Anyang, Gyeonggi, Republic of Korea

8 Department of Neurosurgery, Daejeon Woori Hospital, Daejeon, Gyeonggi, Republic of Korea

9 Department of Neurology, Seoul National University Hospital, Seoul, Republic of Korea

10 Medical Imaging Laboratory, and CyberMed, Inc., Seoul, Republic of Korea

11 Department of Neurosurgery, Seoul National University College of Medicine, Seoul 110-744, Republic of Korea

12 Department of Neurology, Seoul National University College of Medicine, Seoul, Republic of Korea 


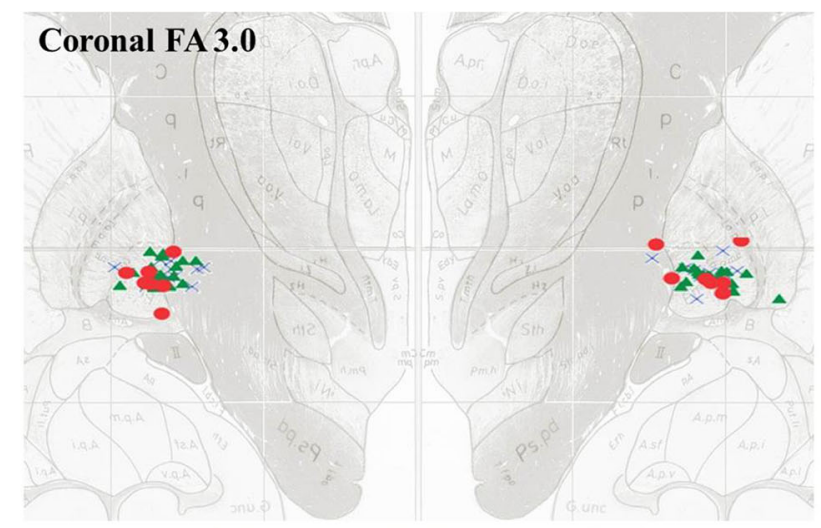

\section{Axial HV -1.5}

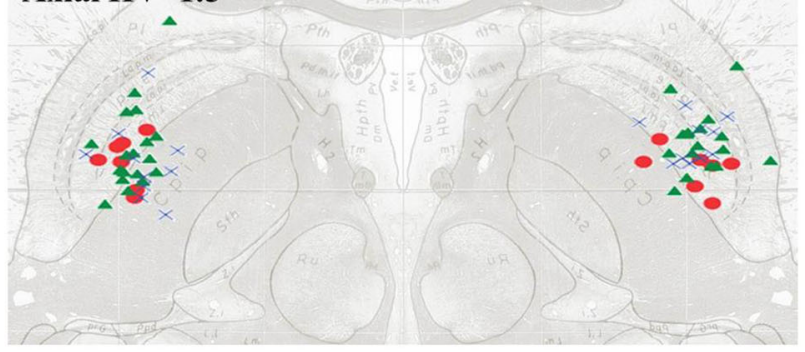

Axial HV -1.5 [Mean electrode location]

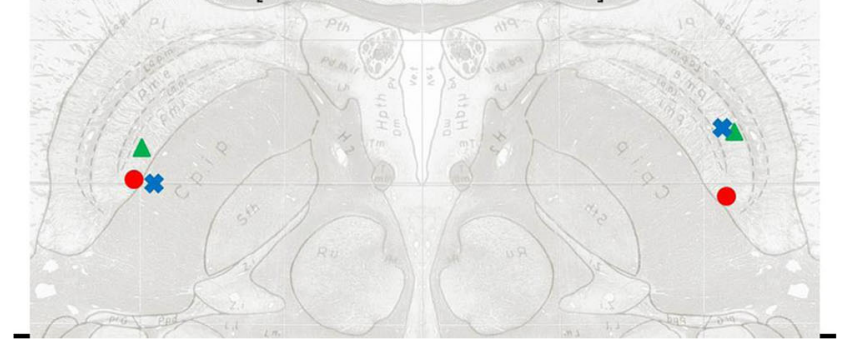

